\title{
The socio-demographic and clinical factors associated with quality of life among patients with brain pathology in a tertiary referral hospital in Malaysia
}

\begin{abstract}
Background: This paper investigates the quality of life of brain pathology patients in relation to their socio-demographic profiles and clinical factors. Methods: This is a cross-sectional study done at a tertiary referral hospital in Kuala Lumpur. A total of 100 patients were recruited in the study after excluding 22 patients who did not met the exclusion criteria. The European Organisation for Research and Treatment of Cancer Quality of Life (EORTC QLQ-C30) selfadministered questionnaire was utilized in the study. The Global health status/QoL, Physical functioning, Role functioning, Emotional functioning, Cognitive functioning, Social functioning, Fatigue, Nausea and vomiting, Pain, Dyspnoea, Insomnia, Appetite loss, Constipation, Diarrhoea, and Financial difficulties were assessed in this study. Results: The most severe impairment in functioning was with lowest score of cognitive functioning (mean score=61) and the most severe symptom was fatigue (mean score=45). There were significant differences in quality of life scores in different socio-demographic groupsand types of brain pathology patients. Patients aged below 40 years old or less had better physical functioning, less symptoms of fatigue and insomnia compared to patients who were more than 40 years old. Male patients faced more financial difficulties compared with female patients. Patients who were married had increased insomnia compared to the single patients. Employed patients had better physical functioning and less financial difficulties compared with patients who were unemployed. Patients who earned > RM 2500.00 monthly had better physical functioning, less symptoms of pain and less financial difficulties than patients who earned $\leq \mathrm{RM}$ 2500.00. Patients with qualifications lower than SPM tended to face more financial difficulties compared to patients with qualifications of SPM or higher. Meningioma patients had better social functioning compared with others, whereas Carvenoma patients had better physical functioning. Meningioma patients had more symptoms of insomnia compared with other patients. All the findings were with $\mathrm{p}$ value less than 0.05. Conclusion: The quality of life of patients with brain pathology is affected by socio-demographic factors and clinical diagnoses. Efforts should be made to improve the overall quality of life of these patients.
\end{abstract}

Keyword : Brain pathology; Quality of life; Socioeconomic factors 